\title{
INVERSION OF INTERVAL VELOCITIES: APPLICATION TO A GEOLOGICAL MODEL FROM A PRE-SALT AREA
}

\author{
Thais Gomes Santana ${ }^{1}$ and Amin Bassrei²
}

\begin{abstract}
Seismic methods study the propagation of elastic wave fields inside the Earth, with the goal to provide subsurface images. In this work, the determination of the time interval velocity distribution is the main information provided. Several synthetic models were used, where one is based in a real situation, a dip section from the pre-salt region, central part of the Santos Basin, Brazil. The methods used to determine interval velocities were based on the Dix transform, singular value decomposition (SVD) and minimum relative entropy (MRE). Dix transform showed excellent results when used in simple geological models, and was coincident to the other two methods. With the addition of a priori information, the SVD and MRE showed to be good methods for the determination of the interval velocities. When comparing SVD and MRE methods the latter showed the best results. When the a priori information is constant, the SVD and MRE methods give the same velocity estimate given from the direct application of the Dix transform.
\end{abstract}

Keywords: inversion of interval velocities, singular value decomposition, minimum relative entropy, pre-salt.

RESUMO. Os métodos sísmicos utilizam o campo de propagação de ondas elásticas no interior da Terra, com o objetivo de fornecer imagens da subsuperfície. Neste trabalho, a determinação do campo de velocidades intervalares é a principal informação a ser fornecida. Foram utilizados modelos sintéticos, sendo um deles baseado em uma situação real, no caso uma sessão dip, na região do pré-sal, parte central da Bacia de Santos. Os métodos usados para determinar as velocidades intervalares foram a transformada de Dix, a decomposição por valores singulares (SVD) e a entropia relativa mínima (MRE). A transformada de Dix, quando usada em modelos geológicos mais simples apresentou excelentes resultados coincidente aos outros dois métodos. Com a adição de estimativas a priori, o SVD e o MRE se mostraram como bons métodos para a determinação das velocidades intervalares, sendo que o MRE apresentou os melhores resultados. Quando a informação a priori é constante, os métodos do SVD e MRE fornecem a mesma estimativa de velocidade que é obtida pela transformada de Dix.

Palavras-chave: inversão de velocidades intervalares, decomposição por valores singulares, entropia relativa mínima, pré-sal.

\footnotetext{
1 Universidade Federal da Bahia, Instituto de Geociências, Rua Barão de Jeremoabo, s/n, Ondina, 40170-115 Salvador, BA, Brazil. Phone: +55(71) 3283-8508 - E-mail: tgs@cpgg.ufba.br

2Universidade Federal da Bahia, Instituto de Geociências, Centro de Pesquisa em Geofísica e Geologia, Instituto Nacional de Ciência e Tecnologia em Geofísica do Petróleo, Rua Barão de Jeremoabo, s/n, Ondina, 40170-115 Salvador, BA, Brazil. Phone: +55(71) 3283-8508 - E-mail: bassrei@ufba.br
} 


\section{INTRODUCTION}

Seismic methods are based on the theory of acoustic and elastic wave propagation in non-homogeneous media. Due to its resolving power the seismic reflection method is the most important geophysical method applied to the oil industry. The main objective of seismic methods is the determination of the interval velocities. The interval velocity is the propagation velocity of the seismic pulse in a given interval in time. For geophysical purposes a geological medium can be described by the distribution of interval velocities, and its determination is done in the last stages of seismic processing. The accurate estimation of the interval velocity field will allow obtaining more reliable seismic images. This topic has great interest in exploration seismology and is discussed in detail in several textbooks, like for instance in Hubral \& Krey (1990).

In this work the interval velocities are estimated using three different methodologies. The first one is the classical Dix transform, and the other two consider the estimation of interval velocities as an inverse problem, where the input data are the pairs of reflection time and RMS velocity. This information is usually available in seismic processing flow at the stage of velocity analysis. In the second method, the inverse problem is solved by generalized inversion implemented by singular value decomposition or SVD. The third method is the minimum relative entropy or MRE, which has a probabilistic principle. These two inversion methods have the advantage to incorporate a priori information, which is not possible in the Dix transform.

In order to test the efficiency, two synthetic models were used, for the inversion of underdetermined problems, where there are more unknowns than equations. In this situation there is a strong need for a priori information. The first model is a 1-D random velocity distribution, with 10 input data and 100 unknowns. The a priori information is effective when it is constant, like for instance when the interval velocity increases linearly from the surface. SVD and MRE provided better results than Dix transform, due to the fact that they can incorporate a non-constant priori information. For this 1-D model, when comparing SVD to MRE, the MRE results were slightly better than SVD.

The second model is a 2-D model inspired in a real situation of the pre-salt geological environment of Santos Basin offshore (Assine et al., 2008). The 2-D model is represented by 1,000 traces, and each trace has 1,000 intervals. Two situations were considered. In the first case, from the interval velocity distribution, for each trace only 20 values of RMS velocity were computed, whereas for the second case, for each trace 50 values of RMS velocity were computed. Again, SVD and MRE provided better results than Dix transform, and when comparing SVD to MRE, the MRE results were slightly better than SVD.

\section{Inverse Problems and Singular Value Decomposition}

We can define a system of $M$ linear equations and $N$ unknowns as:

$$
\left\{\begin{array}{l}
g_{11} m_{1}+g_{12} m_{2}+\cdots+g_{1 N} m_{N}=d_{1} \\
g_{21} m_{1}+g_{22} m_{2}+\cdots+g_{2 N} m_{N}=d_{2}, \\
g_{M 1} m_{1}+g_{M 2} m_{2}+\cdots+g_{M N} m_{N}=d_{M}
\end{array}\right.
$$

where $g_{i j}$ are the coefficients of the linear system, the vector $\boldsymbol{d}$ whose elements are $d_{1}, d_{2}, \ldots, d_{M}$, is the system output, and the vector $\boldsymbol{m}$ whose elements are $m_{1}, m_{2}, \ldots, m_{N}$ is the system solution. In a compact form, the system of equations can be expressed as

$$
\boldsymbol{d}=G \boldsymbol{m} .
$$

In forward modeling procedure, the data parameters are obtained from the model parameters. The opposite happens in the inverse problem, where the estimated model parameters are the solution of the system of linear equations. In other words, we determine the effects of a system where the causes are known (Menke, 1989).

The inverse problem can also be formulated by integral equations, where the unknown function that describes the parameters of the model is part of the integrand. The Fredholm integral equation of the first kind can be used to describe the inverse problem:

$$
d(x)=\int_{a}^{b} G(x, y) m(y) d y,
$$

where $d(x)$ is the function that represents the data, which is known in the inversion process, $G(x, y)$ is the kernel function and depends on two variables, which also must be known and $m(y)$ is a function representing the unknown model parameters.

The solution of Eq. (1) is expressed by:

$$
\boldsymbol{m}=G^{-1} \boldsymbol{d},
$$

if the matrix $G$ is square and has full rank. In general this situation does not happen and the solution can be given by

$$
\boldsymbol{m}=G^{+} \boldsymbol{d},
$$

where $G^{+}$is a $N \times M$ matrix called the generalized inverse or pseudo-inverse.

The singular value decomposition aims to obtain a generalized inverse matrix. The $M \times N$ matrix $G$ can be decomposed as:

$$
G=U \Sigma V^{T},
$$

where $U$ is a $M \times M$ matrix which contains the orthonormalized eigenvectors of $G G^{T}, \sum$ is a $M \times N$ diagonal matrix which contains the square of the eigenvalues of matrix $G^{T} G$, 
and $V$ is a $N \times N$ matrix which contains the orthonormalized eigenvectors of $G^{T} G$. The generalized inverse matrix will be given by

$$
G^{+}=V \Sigma^{+} U^{T} .
$$

\section{Determination of Interval Velocities}

Dix (1955) presented his transform $v_{I N T} \leftrightarrow V_{R M S}$ for a simple model, formed by flat, homogeneous layers. This transform, first suggested by Dürbaum (1954), provides the interval velocities from RMS velocities.

The interval velocity corresponds to the propagation speed of the seismic pulse in a given depth interval. On the other hand RMS (Root Mean Square) velocity can have multiple definitions. According to Dix (1955), it is defined as the inverse of the slope of the tangent line to the curve $T_{n}^{2}(x) \times x^{2}$ at $x=0$, that is,

$$
V_{R M S, n}^{2}=\left[\frac{d T_{n}^{2}(x)}{d x^{2}}\right]_{x \rightarrow 0}^{-1},
$$

where $T(x)$ is the traveltime as function of offset $x$. For any point $x \neq 0$, the RMS velocity is given by:

$$
V_{R M S, n}^{2}(M)=\left[\frac{d T_{n}^{2}(x)}{d x^{2}}\right]_{x \rightarrow M}^{-1} .
$$

Another way to define the $V_{R M S}$ is as the average quadratic interval velocity, $v$, of the layers involved, or

$$
V_{R M S}^{2}(t)=\frac{1}{t} \int_{0}^{t} v^{2}\left(t^{\prime}\right) d t^{\prime} .
$$

In the discrete form the above equation is expressed as:

$$
V_{R M S, n}^{2}=\frac{\sum_{k=1}^{n} v_{k}^{2} t_{k}}{\sum_{k=1}^{n} t_{k}},
$$

and the interval velocity $v_{n}$ as:

$$
v_{n}^{2}=\frac{V_{R M S, n}^{2} T_{0, n}-V_{R M S, n-1}^{2} T_{0, n-1}}{T_{0, n}-T_{0, n-1}},
$$

where $T_{0, n}$ is the reflection traveltime from the surface to $n$-th interface, considering zero offset between source and receiver. $V_{R M S, n}$ is the RMS velocity down to the $n$-th interface. The pair of Eqs. (11) and (12) express the Dix transform (Dix, 1955).

The determination of interval velocities can be expressed as an inverse problem using Eq. (2), and we have that:

$$
d_{j}=T_{j} V_{R M S}^{2}\left(T_{j}\right), \quad j=1, \ldots, M,
$$

and

$$
m_{i}=v_{i}^{2}, \quad i=1, \ldots, N .
$$

Here the $M \times N$ matrix $G$ is an operator from numerical integration of Eq. (10). Using SVD the solution of the inverse problems can be expressed by Eq. (5). The same form of solution is achieved using the least-squares method for the objective function:

$$
\phi(\boldsymbol{m})=\boldsymbol{m}^{T} \boldsymbol{m}+2 \boldsymbol{\lambda}^{T}(\boldsymbol{d}-G \boldsymbol{m}),
$$

where $\boldsymbol{\lambda}$ is the vector of Lagrange multipliers. The minimum of Eq. (15) uses the derivate concept $\partial \phi(\boldsymbol{m}) / \partial \boldsymbol{m}=0$, or:

$$
\frac{\partial \phi(\boldsymbol{m})}{\partial \boldsymbol{m}^{T}}=2 \boldsymbol{m}-2 G^{T} \boldsymbol{\lambda}=0,
$$

to yield

$$
2 \boldsymbol{m}-2 G=G^{T} \boldsymbol{\lambda} .
$$

Substituting Eq. (17) into Eq. (2):

$$
\boldsymbol{d}=G G^{T} \boldsymbol{\lambda},
$$

from where the vector of Lagrange multipliers is obtained as,

$$
\boldsymbol{\lambda}=\left(G G^{T}\right)^{+} \boldsymbol{d} .
$$

Substituting the Eq. (19) into Eq. (17), we have the solution of the inverse problem in the form:

$$
\boldsymbol{m}=G^{T}\left(G G^{T}\right)^{+} \boldsymbol{d} .
$$

The process of inversion through SVD has the advantage of being able to incorporate a priori information, and the previous objective function is modified:

$$
\phi(\boldsymbol{m})=\left(\boldsymbol{m}-\boldsymbol{m}_{0}\right)^{2}+2 \boldsymbol{\lambda}^{T}(\boldsymbol{d}-G \boldsymbol{m}),
$$

where $\boldsymbol{m}_{0}$ is the prior information. Again the minimum of the objective function is achieved by:

$$
\frac{\partial \phi(\boldsymbol{m})}{\partial \boldsymbol{m}^{T}}=2\left(\boldsymbol{m}-\boldsymbol{m}_{0}\right)-2 G^{T} \boldsymbol{\lambda}=0,
$$

to yield

$$
\boldsymbol{m}=G^{T} \boldsymbol{\lambda}+\boldsymbol{m}_{0} .
$$

If we substitute Eq. (23) into Eq. (2), we have that:

$$
\boldsymbol{d}=G\left(G^{T} \boldsymbol{\lambda}+\boldsymbol{m}_{0}\right),
$$

from where the vector of Lagrange multipliers is obtained as:

$$
\boldsymbol{\lambda}=\left(G G^{T}\right)^{+}\left(\boldsymbol{d}-G \boldsymbol{m}_{0}\right) .
$$

Substituting Eq. (25) into Eq. (23), we obtain the solution of the inverse problem as:

$$
\boldsymbol{m}=G^{T}\left(G G^{T}\right)^{+}\left(\boldsymbol{d}-G \boldsymbol{m}_{0}\right)+\boldsymbol{m}_{0} .
$$

Note that if $\boldsymbol{m}_{0}=0$, Eq. (26) reduces to Eq. (20), as expected. 


\section{Minimum Relative Entropy}

Consider a source $m$ emitting $m_{1}, m_{2}, \ldots, m_{N}$ messages with associated probabilities $p_{1}, p_{2}, \ldots, p_{N}$ (where $p_{1}+p_{2}+$ $\left.\cdots+p_{N}=1\right) . I_{i}$ is defined as the information contained in each message $m_{i}$ (Lathi, 1968), and

$$
I_{i}=\log \left(\frac{1}{p_{i}}\right) .
$$

The average information per message emitted by the source is expressed as:

$$
H(m)=\sum_{i=1}^{N} p_{i} I_{i}
$$

where $H(m)$ is denoted the entropy of the system.

Substituting Eq. (27) into Eq. (28) gives:

$$
H(m)=-\sum_{i=1}^{N} p_{i} \log \left(p_{i}\right) .
$$

The entropy of a given source is a function of the message probability. Before the experiment there is an uncertainty for not knowing the results. But, after the experiment, the uncertainty disappears completely when transforming it in gained information (Rietsch, 1988). Since entropy is a measure of uncertainty the probability distribution that generates the maximum uncertainty will have a maximum entropy. This happens when all messages are equally probable, ensuring the highest possible events and, consequently, the maximum uncertainty.

Jaynes (1957) formalized the concept of maximum entropy, and also examined the relationships between statistical mechanics and information theory. In the absence of a priori information, he proposed a different view on this issue, where he states that the maximum entropy estimate is the less biased of a given information. Because there is insufficient knowledge to determine which event is most likely to happen, we consider that the probabilities of all events are equal. Therefore, the maximization of the entropy is a methodology of logical reasoning that ensures no usage of any inconsistent ideas on the problem of prediction. The principle of maximum entropy is applicable to problems with incomplete data, may or may not also involve a repetitive situation like a probabilistic experiment (Jaynes, 1982).

The principle of minimum relative entropy, or MRE, was developed in the field of statistics by Kullback \& Leibler (1951). The amount of information, $I$, obtained in the transmission of a message can be expressed as:

$$
I=\log \left[\frac{q(x)}{p(x)}\right],
$$

where $p(x)$ is the prior probability density function (PDF), or the likelihood that a given message was sent; and $q(x)$ is the posterior PDF, or the probability of such a message arrives to the recipient. For the continuous case the relative entropy is defined as:

$$
H(q, p)=\int q(x) \log \left[\frac{q(x)}{p(x)}\right] d x .
$$

The application of MRE in inverse problems is made using the Fredholm integral equation of the first kind (Ulrych et al., 1990):

$$
d_{j}=\int_{a}^{b} H_{j}(u) m(u) d u, \quad j=1, \ldots, M,
$$

where $d_{j}$ are the known data, $H_{j}(u)$ is the known kernel, and $m(u)$ is the unknown model parameters. Our objective is to obtain a final estimate of $m$, denoted by $\tilde{m}$ which satisfies Eq. (32). The discrete version of Eq. (32) is written as:

$$
d_{j}=\sum_{n=0}^{N} H_{j}\left(u_{n}\right) \tilde{m}\left(u_{n}\right) \Delta u, \quad j=1, \ldots, M,
$$

where $u_{n}$ and $\Delta u$ are defined as:

$$
\begin{aligned}
& u_{n}=(b-a)\left(\frac{n}{N}\right)+a, \\
& \Delta u=u_{n+1}-u_{n}=\frac{b-a}{N} .
\end{aligned}
$$

We can write the discrete Fredholm equation as:

$$
\frac{d_{j}}{\Delta u}=\sum_{n=0}^{N} H_{j}(n) \tilde{m}(n), \quad j=1, \ldots, M,
$$

where $u$ is a function of $n$. The vector $\tilde{m} d(n)$ is an estimate of $m(n)$ as:

$$
\tilde{m}(n)=\int_{0}^{\infty} m(n) q(\boldsymbol{m}) d \boldsymbol{m} .
$$

Substituting Eq. (37) into Eq. (36) gives:

$$
\begin{gathered}
\frac{d_{j}}{\Delta u}=\int_{0}^{\infty} q(\boldsymbol{m})\left[\sum_{n=0}^{N} H_{j}(n) m(n)\right] d \boldsymbol{m}, \\
j=1, \ldots, M
\end{gathered}
$$

where $q(\boldsymbol{m})$ is the PDF associated with $\boldsymbol{m}$. Consider an initial estimate of $m(n)$ in the inversion process, denoted by $s(u)$, that corresponds to the a priori information to be used as an input data of the inverse problem. According to Shore (1981), it is assumed that $s(n)$ are expected values of some PDF. 
So we choose this initial PDF, expressed as $p(m)$, as having minimum relative entropy in relation another PDF which is constant. The initial PDF proposed by Shore (1981) is:

$$
p(\boldsymbol{m})=\prod_{n=o}^{N} \frac{1}{s(n)} e^{\left[-\frac{m(n)}{s(n)}\right]} .
$$

We will minimize the entropy of $q(\boldsymbol{m})$ with respect to $p(\boldsymbol{m})$, taking into account the constraint given by Eq. (38), and the normalization constraint applied to $q(\boldsymbol{m})$ as:

$$
\int_{0}^{\infty} q(\boldsymbol{m}) d \boldsymbol{m}=1
$$

The objective function is constructed as:

$$
\begin{gathered}
\phi(\boldsymbol{m})=\int_{0}^{\infty} q(\boldsymbol{m}) \log \left[\frac{q(\boldsymbol{m})}{p(\boldsymbol{m})}\right] d \boldsymbol{m} \\
+\mu\left[\int_{0}^{\infty} q(\boldsymbol{m}) d \boldsymbol{m}-1\right]+\sum_{j=1}^{M} \lambda_{j} \\
\times\left[\int_{0}^{\infty} q(\boldsymbol{m}) \sum_{n=0}^{N} H_{j}(n) m(n) d \boldsymbol{m}-\frac{d_{j}}{\Delta u}\right] .
\end{gathered}
$$

The first term of Eq. (41) is the relative entropy integral. Taking the derivate of $\phi(\boldsymbol{m})$ with respect to $\boldsymbol{m}$ and equaling to zero,

$$
\begin{aligned}
0= & \int_{0}^{\infty}\left\{\log \left[\frac{q(\boldsymbol{m})}{p(\boldsymbol{m})}\right]+1+\mu\right. \\
& \left.+\sum_{j=1}^{M} \lambda_{j} \sum_{n=0}^{N} H_{j}(n) m(n)\right\} d \boldsymbol{m},
\end{aligned}
$$

Reversing the summation order in Eq. (42), making $c=$ $\exp (-1-\mu)$, and writing in terms of the multiplicand, we obtain:

$$
\begin{gathered}
q(\boldsymbol{m})=c p(\boldsymbol{m}) \prod_{n=0}^{N} \exp \\
\times\left[-m(n) \sum_{j=1}^{M} \lambda_{j} H_{j}(n)\right] .
\end{gathered}
$$

Substituting the initial PDF, given in the Eq. (39) into Eq. (43), we have that:

$$
\begin{gathered}
q(\boldsymbol{m})=c \prod_{n=0}^{N} \frac{1}{s(n)} \exp \\
\times\left\{-m(n)\left[\frac{1}{s(n)}+\sum_{j=1}^{M} \lambda_{j} H_{j}(n)\right]\right\} .
\end{gathered}
$$

Applying the normalization constraint in Eq. (40) into Eq. (44):

$$
\begin{gathered}
q(\boldsymbol{m})=c \prod_{n=0}^{N} \frac{1}{s(n)} \int_{0}^{\infty} \exp \\
\times\left\{-m(n)\left[\frac{1}{s(n)}+\sum_{j=1}^{M} \lambda_{j} H_{j}(n)\right]\right\} d \boldsymbol{m}=1 .
\end{gathered}
$$

Solving the integral in Eq. (45) we obtain an equation for $c$ :

$$
c=\prod_{n=0}^{N}\left[\frac{1}{s(n)}+\sum_{j=1}^{M} \lambda_{j} H_{j}(n)\right] .
$$

Substituting Eq. (46) into Eq. (45), we have that:

$$
\begin{aligned}
& q(\boldsymbol{m})=\prod_{n=0}^{N}\left[\frac{1}{s(n)}+\sum_{j=1}^{M} \lambda_{j} H_{j}(n)\right] \exp \\
& \times\left\{-m(n)\left[\frac{1}{s(n)}+\sum_{j=1}^{M} \lambda_{j} H_{j}(n)\right]\right\},
\end{aligned}
$$

where $q(\boldsymbol{m})$ is the posterior PDF estimate. Substituting Eq. (47) into Eq. (37), we obtain:

$$
\begin{aligned}
& \tilde{m}(n)=\int_{0}^{\infty} m(n) \prod_{n=0}^{N}\left[\frac{1}{s(n)}+\sum_{j=1}^{M} \lambda_{j} H_{j}(n)\right] \exp \\
& \times\left\{-m(n)\left[\frac{1}{s(n)}+\sum_{j=1}^{M} \lambda_{j} H_{j}(n)\right]\right\} d \boldsymbol{m} .
\end{aligned}
$$

Making a change of variable in the terms independent of $m$ :

$$
\alpha=\frac{1}{s(n)}+\sum_{j=1}^{M} \lambda_{j} H_{j}(n),
$$

to give (48) in the form

$$
\tilde{m}(n)=\int_{0}^{\infty} m(n) \prod_{n=0}^{N} \alpha \exp [-m(n) \alpha] d \boldsymbol{m} .
$$

Performing integration by parts, we arrive at:

$$
\begin{aligned}
\tilde{m}(n)= & \frac{1}{\frac{1}{s(n)}+\sum_{j=1}^{M} \lambda_{j} H_{j}(n)}, \\
& (n=0, \ldots, N),
\end{aligned}
$$

where, $\tilde{m}(n)$ is the MRE model parameters estimate, $s(n)$ is a priori model to be used in the inversion, $H_{j}(n)$ are the kernel matrix elements of the forward problem, $\lambda_{j}$ are the Lagrange multipliers, which are associated to the constraints in the minimization problem. 


\section{RESULTS}

The proposed methodologies to estimate interval velocity distributions are applied to one 1-D model with 100 layers, and 2D model with 1000 layers representing a pre-salt model based on real data displayed in Assine et al. (2008). Initially, the Dix transform is applied directly to the RMS velocity data, and then compared to the results obtained by the SVD and the MRE inversions.

The performance of these methods was evaluated using the criterion of relative RMS deviation $\left(\epsilon_{R M S}\right)$ defined as:

$$
\epsilon_{R M S}=\frac{\sqrt{\sum_{i=1}^{N}\left(v_{i}^{\text {true }}-v_{i}^{\text {est }}\right)^{2}}}{\sqrt{\sum_{i=1}^{N}\left(v_{i}^{\text {true }}\right)^{2}}},
$$

where $v^{\text {true }}$ is the vector of true velocities, and $v^{e s t}$ is the vector of estimated velocities. This measure is global, that is, for the all dataset, and can be computed for the three approaches (Dix, SVD and MRE). In the case of Dix transform:

$$
\epsilon_{D I X}=\frac{\sqrt{\sum_{i=1}^{N}\left(v_{i}^{\text {true }}-v_{i}^{\text {dix }}\right)^{2}}}{\sqrt{\sum_{i=1}^{N}\left(v_{i}^{\text {true }}\right)^{2}}} .
$$

For the SVD method:

$$
\epsilon_{S V D}=\frac{\sqrt{\sum_{i=1}^{N}\left(v_{i}^{\text {true }}-v_{i}^{\text {svd }}\right)^{2}}}{\sqrt{\sum_{i=1}^{N}\left(v_{i}^{\text {true }}\right)^{2}}} .
$$

And for the MRE:

$$
\epsilon_{M R E}=\frac{\sqrt{\sum_{i=1}^{N}\left(v_{i}^{\text {true }}-v_{i}^{\text {mre }}\right)^{2}}}{\sqrt{\sum_{i=1}^{N}\left(v_{i}^{\text {true }}\right)^{2}}} .
$$

The first computational model has 100 layers with different interval velocities. In this case, only 10 values of RMS velocities are computed, the first value at the base of the 10-th layer, the second at the base of 20-th layer, and so on, according to the diagram of Figure 1. This characterizes an underdetermined problem ( $M=10$ and $N=100$ ) where the a priori information has an important role.

Figure 2 shows the interval velocity curve in red for a situation of complex geological environment with multiple velocity reversions. The $V_{R M S}$ curve is in black.

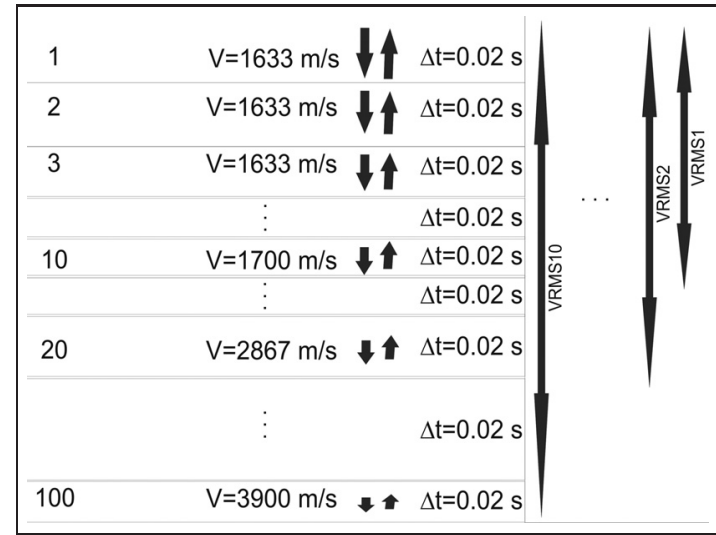

Figure 1 - Model with 100 horizontal plane layers with uniform time intervals, where only 10 values of $V_{R M S}$ are calculated.

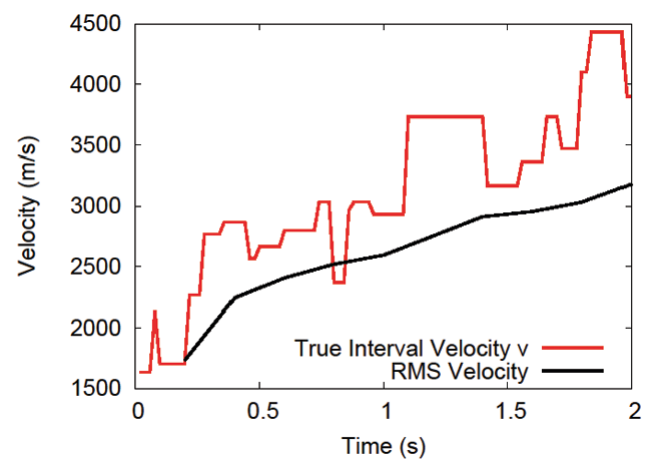

Figure 2 - Model with 100 horizontal plane layers, where only 10 values of RMS velocity are calculated. The true time interval velocities $v_{T R U E}$ are in red and the $v_{R M S}(t)$ in black.

Figure 3 shows the inversion using the $V_{R M S}$ as input. The true interval velocities are in red, the estimated interval velocities by Dix transform in squares, and the estimated interval velocities by SVD in black dots. No a priori information was used and the $\epsilon_{D I X}$ was equal to the $\epsilon_{S V D}\left(\epsilon_{D I X}=\epsilon_{S V D}=6.68 \%\right)$.

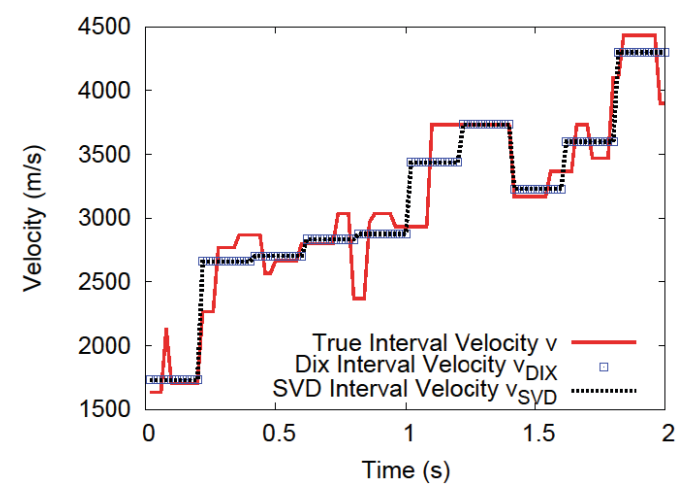

Figure 3 - Inversion without a priori information. The $v_{T R U E}$ are in red, the $v_{D I X}$ are in blue squares and the $v_{S V D}$ are in black dots.

The interval velocity model were then calculated with the possibility of incorporating a priori information. The same a priori information was used for both methods (SVD and MRE). 
Figure 4 shows the result for a priori information constant and equal to $2,000 \mathrm{~m} / \mathrm{s}$, which has $41.17 \%$ of $\epsilon_{R M S}$ in relation to the true velocities. The $\epsilon_{R M S}$ was the same for the three methods; that is, $\epsilon_{D I X}=\epsilon_{S V D}=\epsilon_{M R E}=6.68 \%$.

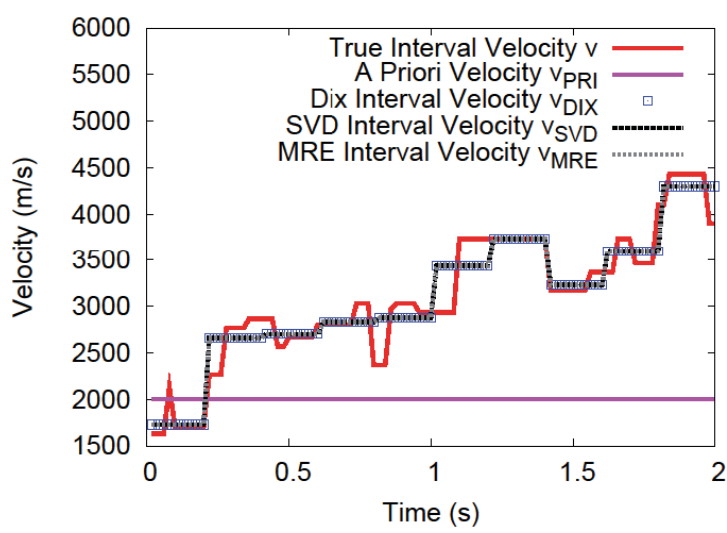

Figure 4 - Inversion with a priori information constant and equal to 2,000 m/s. The $v_{T R U E}$ are in red, the a priori information is in purple, the $v_{D I X}$ are in blue squares, the $v_{S V D}$ are in black dots, and the $v_{M R E}$ are in gray dots.

Figure 5 shows the results with a priori information with a linearly increasing velocity, and a variation of $10 \mathrm{~m} / \mathrm{s}$ in each interval, which has $24.20 \%$ of $\epsilon_{R M S}$ in relation to the true velocities. In this case $\epsilon_{S V D}=6.46 \%$ and $\epsilon_{M R E}=6.32 \%$ was slightly better. Notice that the $\epsilon_{D I X}$ does not change because it does use a priori information; that is, $\epsilon_{D I X}=6.68 \%$.

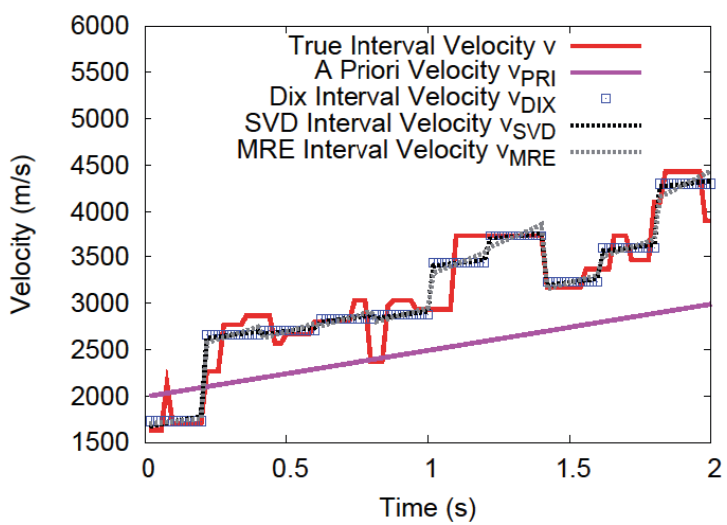

Figure $\mathbf{5}$ - Inversion with a linearly increasing prior information, from 2,000 to $3,000 \mathrm{~m} / \mathrm{s}$; the velocity increases $10 \mathrm{~m} / \mathrm{s}$ in each layer. The $v_{T R U E}$ are in red, the $v_{P R I}$ are in purple, the $v_{D I X}$ are in blue squares, the $v_{S V D}$ are in black dots, and the $v_{M R E}$ are in gray dots.

Figure 6 shows the results when the a priori velocity increases $25 \mathrm{~m} / \mathrm{s}$ in each interval. In this case, the $\epsilon_{R M S}$ was $11.75 \%$. Here $\epsilon_{S V D}=6.29 \%$ and $\epsilon_{M R E}=6.19 \%$. Again, $\epsilon_{D I X}=6.68 \%$. The results are good, considering the fact that the true model has a random pattern. Comparing the three methods the $\epsilon_{M R E}$ is the lowest, followed by the $\epsilon_{S V D}$ and then by the $\epsilon_{D I X}$.

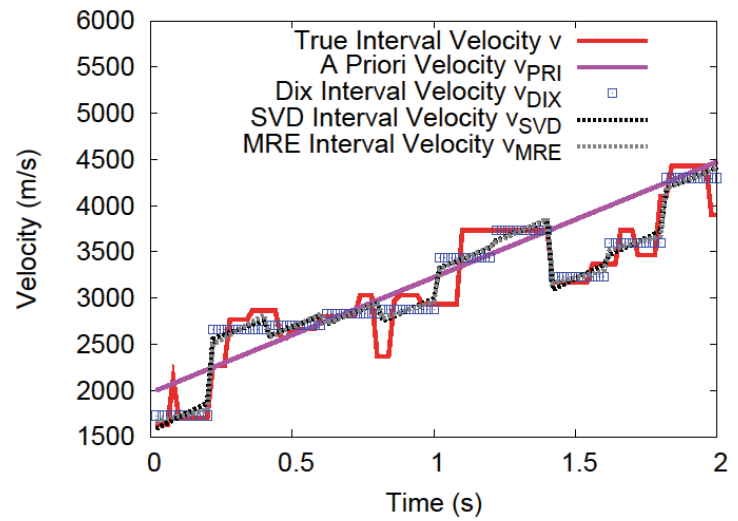

Figure $\mathbf{6}$ - Inversion with a linearly increasing prior information $v_{P R I}$, from 2,000 to $4,500 \mathrm{~m} / \mathrm{s}$; the velocity increases $25 \mathrm{~m} / \mathrm{s}$ in each interval. The $v_{T R U E}$ are in red, the $v_{P R I}$ are in purple, the $v_{D I X}$ are in blue squares, the $v_{S V D}$ are in black dots, and the $v_{M R E}$ are in gray dots.

The model shown in Figure 7, is a situation with a pre-salt geology, inspired on a dip section of the central part of Santos Basin (right portion of Fig. 8 of Assine et al., 2008). The sea floor curves gently and the next reflector in the model is also not horizontal and with velocity of $2,200 \mathrm{~m} / \mathrm{s}$. In the model the velocities range from 1,500 to $6,200 \mathrm{~m} / \mathrm{s}$. The reflection time is displayed in the vertical axis, and ranges from 0 to $7,770 \mathrm{~ms}$, and the model has a horizontal length of $44,640 \mathrm{~m}$.

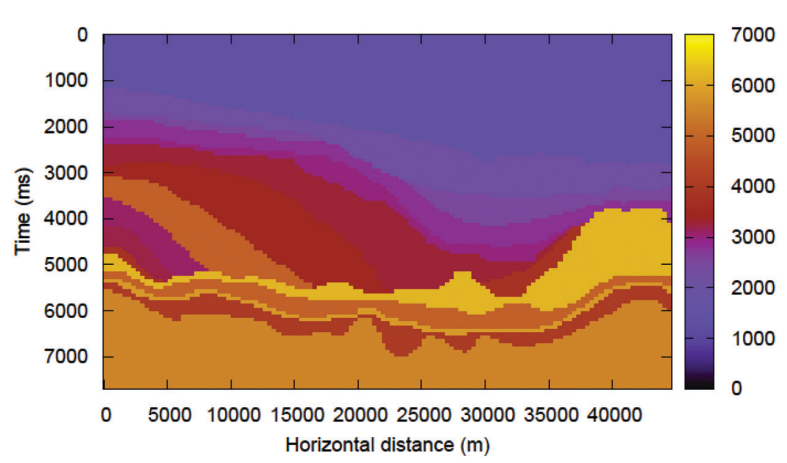

Figure 7 - Two-dimensional true model with a pre-salt situation, based on information contained in Assine et al. (2008). The color bar indicates the $v_{T R U E}$ in $\mathrm{m} / \mathrm{s}$.

The 2-D model is represented by 1,000 traces, and each trace has 1,000 intervals. From the interval velocity distribution, for each trace only 20 values of $v_{R M S}$ were computed, being the first value to the base of the 50-th layer, the second value at the base of 100-th layer, and so on. Figure 8 shows this $v_{R M S}$ distribution. We also considered another situation, where for each trace 50 values of $v_{R M S}$ were computed, being the first value to the base of the 20-th layer, the second value at the base of 40-th layer, and so on. Figure 8 shows this $v_{R M S}$ distribution. 


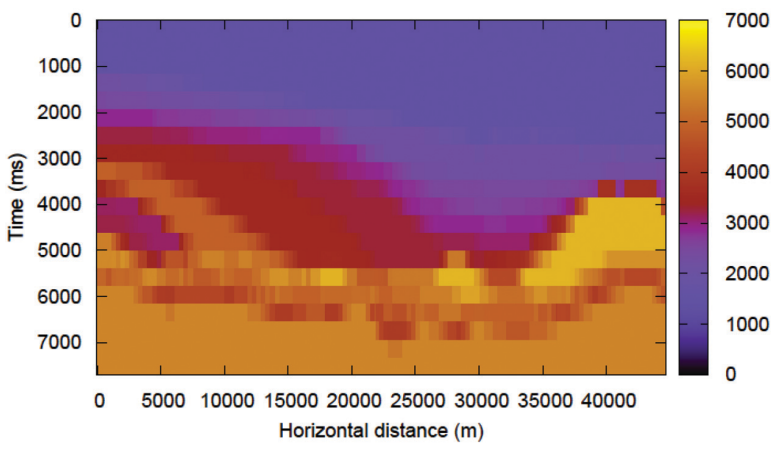

Figure 8 - Two-dimensional $v_{S V D}$ and $v_{M R E}$ distributions with 20 values of $v_{R M S}$, and with a linearly increasing prior information. The color bar indicates the $v_{R M S}$ in $\mathrm{m} / \mathrm{s}$.

The inversions without a priori information will not be showed, since the two methods gave the same result: $\epsilon_{D I X}=$ $\epsilon_{S V D}=9.22 \%$ when the input data were 20 values of $v_{R M S}$ and $\epsilon_{D I X}=\epsilon_{S V D}=5.87 \%$ when the input data were 20 values of $v_{R M S}$.

Two a priori velocity distribution were used for the SVD and MRE inverse methods: constant and equal to $2,000 \mathrm{~m} / \mathrm{s}$; linearly increasing prior information, from 2,000 to $6,000 \mathrm{~m} / \mathrm{s}$; and linearly increasing prior information, from 1,500 to $6,500 \mathrm{~m} / \mathrm{s}$, the velocity increases $5 \mathrm{~m} / \mathrm{s}$ in each interval. The $\epsilon_{P R I}$ were, respectively, $57.37 \%$ and $24.89 \%$.

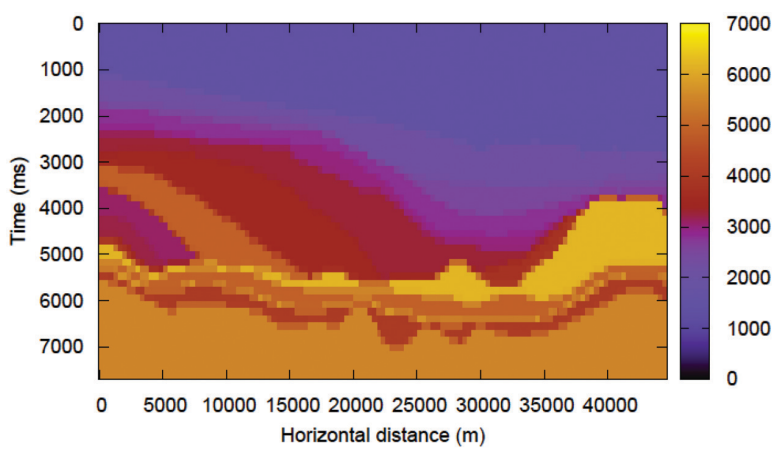

Figure 9 - Two-dimensional $v_{S V D}$ and $v_{M R E}$ distributions with 50 values of $v_{R M S}$, and with a linearly increasing prior information. The color bar indicates the $v_{R M S}$ in $\mathrm{m} / \mathrm{s}$.

The two a priori models were used in the SVD and MRE inversions. For the constant a priori information the result was the same, that is, $\epsilon_{S V D}=\epsilon_{M R E}=5.87 \%$ for 50 values of $v_{R M S}$, and $\epsilon_{S V D}=\epsilon_{M R E}=9.22 \%$ for 50 values of $v_{R M S}$.

For the second a priori information (velocity increase of $5 \mathrm{~m} / \mathrm{s}$ for each interval), for the case with 20 values of of $v_{R M S}$, the SVD result improved a little $\left(\epsilon_{S V D}=9.14 \%\right)$, and the MRE output was a little better that SVD $\left(\epsilon_{M R E}=9.12 \%\right)$. This small difference between SVD and MRE is not noticeable in the images, in such a way that we are only presenting one image for both methods (Fig. 10).

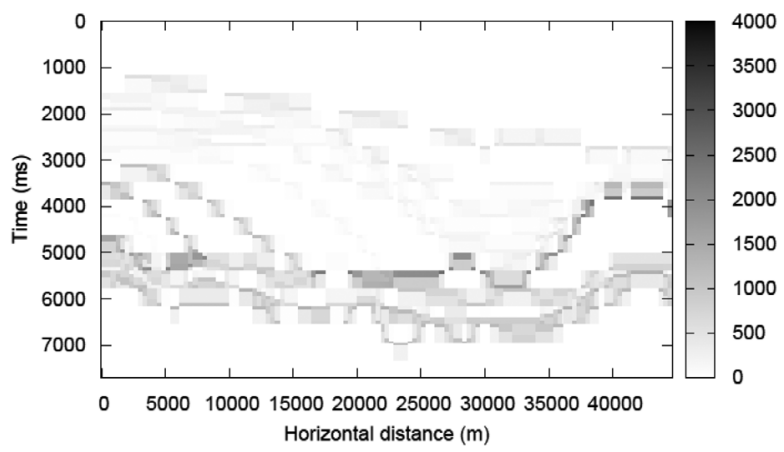

Figure 10 - Two-dimensional residual between $v_{T R U E}$ and the $v_{M R E}$ (or $v_{S V D}$ ) with 20 values of $v_{R M S}$, and with a linearly increasing prior information. The color bar indicates the residual in $\mathrm{m} / \mathrm{s}$.

Figure 11 shows the result for the second a priori information (velocity increase of $5 \mathrm{~m} / \mathrm{s}$ for each interval), for the case with 50 values of of $v_{R M S}$, the SVD and MRE results were the same $\left(\epsilon_{S V D}=\epsilon_{M R E}=5.85 \%\right)$.

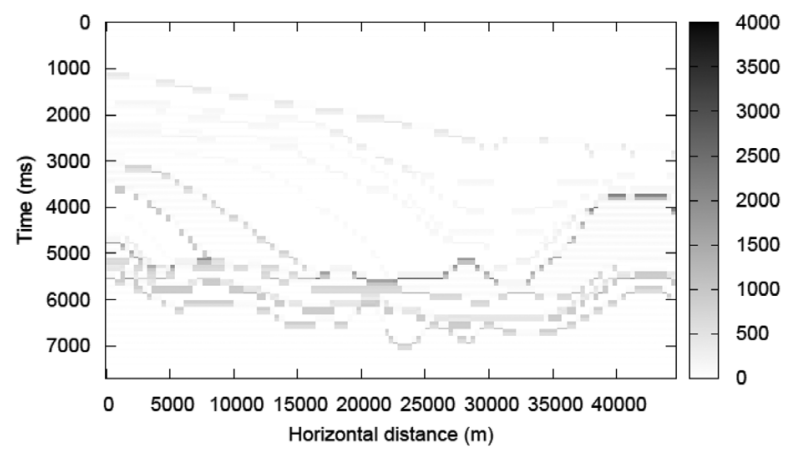

Figure 11 - Two-dimensional residual between $v_{T R U E}$ and the $v_{M R E}$ (or $v_{S V D}$ ) for 50 values of $v_{R M S}$, and with a linearly increasing prior information. The color bar indicates the residual in $\mathrm{m} / \mathrm{s}$.

In order to evaluate better the effectiveness of the methodology in the 2-D example we obtain the residual velocities by subtracting the estimated interval velocity distribution from the true interval velocity distribution. Figure 10 shows the velocity residual when the input data had 20 values of RMS velocity and with a linearly increasing prior information. Figure 11 shows the velocity residual for the same prior information when the input data had 50 values of RMS velocity.

\section{CONCLUSIONS}

The use of the Dix transform to obtain estimates of the interval velocity field shows limitations, and it is feasible to only simple geological models. The SVD and MRE approaches are technically different: SVD is deterministic, and MRE is probabilistic, 
and for underdetermined problems (more unknowns than equations), they need a priori information. For a random 1-D model the SVD and MRE methods provided better results, due to the fact that they can incorporate a priori information. The second model was 2-D, but the inverse approach was applied in a 1-D manner, that it, trace by trace. After the inversion the 1-D interval velocities distributions were rearranged in a 2-D display. Also for this second model the MRE provided better results than SVD, when a non-constant prior information was used.

\section{ACKNOWLEDGMENTS}

T.G. Santana thanks ANP and CNPq for a M.Sc. scholarship. A. Bassrei would like to thank CNPq and PETROBRAS for the project National Institute of Science and Technology in Petroleum Geophysics (INCT-GP), CNPq for the project 308690/2013-3 (research fellowship), and FINEP for sponsoring the CT-PETRO Network in Exploration Geophysics (Rede 01).

\section{REFERENCES}

ASSINE ML, CORREEA FS \& CHANG HK. 2008. Depocenter migration in the Santos Basin: importance in hydrocarbon exploration (in Portuguese). Revista Brasileira de Geociências, 38: 111-127.

DIX CH. 1955. Seismic velocities from surface measurements. Geophysics, 20: 68-86.
DÜRBAUM H. 1954. Zur Bestimmung von Wellengeschwindigkeiten aus Reflexionsseismischen Messungen. Geophysical Prospecting, 2: $151-167$.

HUBRAL P \& KREY T. 1980. Interval Velocities from Seismic Reflection Time Measurements. Society of Exploration Geophysicists, Tulsa, $203 \mathrm{pp}$.

JAYNES ET. 1957. Information theory and statistical mechanics. Physical Review, 106: 620-630.

JAYNES ET. 1982. On the rationale of maximum-entropy methods. Proceedings of the Institute of Electrical and Electronics Engineers, 70: 939-952.

KULLBACK S \& LEIBLER RA. 1951. On information and sufficiency. Annals of Mathematical Statistics, 22: 79-86.

LATHI BP. 1968. Communication Systems. John Wiley, New York, $446 \mathrm{pp}$.

RIETSCH E. 1988. The maximum entropy approach to the inversion of one-dimensional seismograms. Geophysical Prospecting, 36: 365-382.

SHORE JE. 1981. Minimum cross-entropy spectral analysis. Institute of Electrical and Electronics Engineers Transactions on Acoustics, Speech and Signal Processing, 29: 230-237.

ULRYCH T, BASSREI A \& LANE M. 1990. Minimum relative entropy inversion of $1 \mathrm{D}$ data with applications. Geophysical Prospecting, 38: 465-487.

Recebido em 11 dezembro, 2013 / Aceito em 8 janeiro, 2015

Received on December 11, 2013 / Accepted on January 8, 2015

\section{NOTES ABOUT THE AUTHORS}

Thais Gomes Santana holds a B.Sc. degree in Geophysics (2009) and an M.Sc. in Geophysics (2013), both from the Universidade Federal da Bahia (UFBA). Geophysicist at GEORADAR from 2011 to 2012 and is currently a CNPq fellow at UFBA working on shallow marine seismics and ground penetrating radar. Experience in seismic acquisition, processing, interpretation and inversion.

Amin Bassrei holds a B.Sc. degree in Electrical Engineering (1985) and a D.Sc. degree in Geophysics (1990), both from the Universidade Federal da Bahia (UFBA). Post-doctoral training in Petroleum Geophysics at MIT's Earth Resources Laboratory (1992-1993). Faculty at UFBA since 1995 and is currently associate professor IV at the Department of Geophysics and researcher at the Research Center in Geophysics and Geology (CPGG/UFBA). Undergraduate coordinator (2004-2008) and graduate coordinator (2009-2011, 2013-2015) in the Geophysics Program at UFBA. Since 2009 is coordinator of the CT-PETRO Research Network in Exploration Geophysics - FINEP Rede 01. Experience in exploration seismology, seismic and gravity inversion, seismic tomography, signal analysis, and $\mathrm{CO}_{2}$ geological storage. 